\title{
Población extranjera y turismo residencial en el litoral de Alicante (1960-2011): repercusiones territoriales $^{1}$
}

\author{
Álvaro Francisco Morote. Universidad de Alicante, Alicante, España. \\ María Hernández. Universidad de Alicante, Alicante, España.
}

RESUMEN | El litoral de la provincia de Alicante ha registrado importantes cambios territoriales desde la década de 1960, como consecuencia de la difusión de las actividades turísticas. Las transformaciones territoriales, entre las que destaca la configuración de una dorsal de poblamiento litoral, se completan con las sociales. La llegada de población extranjera jubilada o prejubilada procedente del norte y centro de Europa ha hecho de la provincia de Alicante (España) uno de los territorios donde el turismo residencial tiene mayor peso a escala nacional. El objetivo de esta investigación es, por un lado, analizar los factores que justifican el asentamiento de amplios colectivos de inmigrantes en estas tierras y, por otro, poner de manifiesto las repercusiones sociales y territoriales asociadas a su llegada. La realización de entrevistas en urbanizaciones de municipios litorales y prelitorales permite avanzar en el conocimiento de estos factores, más allá de los datos estadísticos.

Palabras Clave | litoral de Alicante, turismo residencial, población extranjera.

ABSTRACT | The coast of the province of Alicante has undergone significant territorial changes since the 1960's as a result of the development of tourism activities, where territorial changes, like the shaping of coastal settlement, are met with social ones. The arrival of retired or pre-retired foreign population from Northern and Central Europe makes the province of Alicante (Spain) one of the Mediterranean regions where residential tourism has acquired greatest relevance at a national level. The aim of this research is, on the one hand, to analyze the factors that explain the settlement of large groups of immigrants in this area, and, on the other hand, to highlight social and territorial impacts associated with their arrival. A survey conducted in the residential areas of coastal municipalities allowed moving forward in the knowledge of these factors, beyond plain statistical data.

KEY WORDS | coast of Alicante, residential tourism, foreign population.

Recibido el 26 de mayo de 2014, aprobado el 24 de julio de 2015

E-mail: A.Morote, alvaro.morote@ua.es|M.Hernández, maria.hernandez@ua.es

1 Este artículo es resultado de la concesión de una beca predoctoral de Formación de Profesorado Universitario del Programa Nacional de Investigación Científica, Desarrollo e Innovación Tecnológica (FPU). Se inserta, asimismo, en el Proyecto de Investigación "Urbanización y metabolismo hídrico en el litoral de Alicante: análisis de tendencias para el período 2000-2010" (CSO2OI 2-36997-CO2-02), financiado por el Ministerio de Economía y Competitividad. 


\section{Introducción}

El litoral mediterráneo español, y el de la provincia de Alicante (Comunidad Valenciana), han registrado notables transformaciones sociales, territoriales y económicas en las últimas décadas. Durante la segunda mitad del pasado siglo y primeros ańos del presente, la Comunidad Valenciana experimentó un fuerte desarrollo socioeconómico vinculado al impulso de las funciones urbanas y como resultado de la difusión de nuevas actividades (turismo, comercio e industria). Los procesos de urbanización, industrialización y desarrollo turístico han sido factor determinante para la concentración del $77 \%$ de la población valenciana en una dorsal de poblamiento que se prolonga desde la plana costera de Vinaroz-Benicarló, al norte de Castellón, hasta Pilar de la Horadada, en la costa sur de Alicante (Rico \& Hernández, 2008).

A partir de la década de 1960, el proceso de apertura y diversificación económica impulsado desde el gobierno contempló el turismo como un sector fundamental; y vinculada a este, la industria inmobiliaria (Pack, 2006), basada en un primera fase en la construcción de plantas hoteleras y, posteriormente, de alojamientos residenciales con finalidad turística. Este proceso vino a su vez acompañado de un fuerte desarrollo turístico y residencial, que ha situado a la Comunidad Valenciana entre las regiones con una mayor proyección turística de España. Algunos datos corroboran esta afirmación. En 2012, recibió 22,2 millones de visitantes que sumaron 153,9 millones de pernoctaciones, cifra que la situó como tercer destino por detrás de Andalucía y Cataluña. La oferta de alojamiento ascendía a 380.970 plazas (Agencia Valenciana de Turismo, 2013a). Las 124.076.154 plazas hoteleras, que se concentran en un 50\% en la provincia de Alicante, acogieron 6,93 millones de viajeros y generaron algo más de 24 millones de pernoctaciones, lo que supuso el 8,6\% del total nacional (Agencia Valenciana de Turismo, 2013b). Ascendió al $10,2 \%$ la población activa valenciana vinculada al sector turístico y a un $12,6 \%$ su aportación al PIB regional en 2010, último año con datos disponibles (Exceltur, 2011). Esta oferta se completa con la residencial, integrada por viviendas y apartamentos no reglados, que sumaban, a principios de este siglo, alrededor de 2,2 millones de plazas (Vera, Casado \& Ramón, 2004) y representaban el 20\% de la oferta total espańola. La oferta turística residencial es la tipología de alojamiento dominante, y pone de manifiesto el enorme peso del turismo residencial y del sector inmobiliario en esta región.

El proceso más espectacular ha sido, no obstante, el notable incremento de la segunda residencia. En el intercensal de 1991 a 2001, el número total de viviendas en la Comunidad Valenciana habría pasado de 2.094.033 a 2.547.775, es decir, un saldo positivo de 453.742 unidades (Hernández, Rico \& Juárez, 2010). En el período entre 2001 y 2011, la Valenciana era la quinta región española con mayor incremento del parque de viviendas (un 23,5\% frente a la media nacional, que se situó en el 20,3\%). El porcentaje de residencia secundaria en 2001 se encontraba en torno al $22 \%$ del total de viviendas y se elevaría hasta el $40 \%$ si se incluyera la vacía, frente al 15\% de 1991 y el escaso 3\% de 1960, valores que se han mantenido en el intercensal 2001-2011. Estas cifras se incrementan significativamente al descender a 
la escala local: prácticamente en la totalidad de los municipios litorales y prelitorales de la provincia de Alicante, el porcentaje de vivienda no principal supera el 50\%. Tal valor supera la media provincial, situada en el 26,9\% (INE, 2012).

La dinámica descrita se relaciona con la intensa actividad de la construcción que se ha desarrollado en el país, y especialmente en la fachada mediterránea, desde mediados de la década de los noventa y hasta 2008. El número total de viviendas en España se incrementó en casi cinco millones entre los ańos 2001 y 2011 (Ministerio de Fomento, 2012), aproximadamente un 25\%, al pasar el parque de viviendas de 21,03 a 26,01 millones, respectivamente. Este porcentaje se concentra mayoritariamente entre 2001 y 2008, coincidiendo con el boom de la construcción, tendencia que se ralentiza a partir de 2007, cuando se inicia la actual crisis inmobiliaria. Este rápido desarrollo urbano presenta una marcada concentración territorial. Por regiones, Andalucía (1.115.659 viviendas), Cataluña (769.786), Comunidad Valenciana (791.882), Murcia (292.708) y Baleares (118.069) acumulan más de 3 millones de certificaciones de fin de obra, lo que supone aproximadamente el $55 \%$ del total nacional. La Valenciana, donde se inserta la provincia de Alicante, por ejemplo, entre 1997 y 2008 fue la tercera comunidad autónoma en número de viviendas iniciadas en esa década. En este período se construyeron unas 790.000 unidades residenciales, lo que significó un incremento del $36 \%$ con respecto a las existentes en 1996 (Burriel, 2008), una concentración regional que se acentúa a escala provincial. Las once provincias ribereñas del Mediterráneo español suman 2.487.262 nuevas viviendas visadas para el período 2000-2011, es decir, el 43,88\% del total estatal (cincuenta provincias). Sorprendente resulta esta actividad en la provincia de Alicante, que se situó en el tercer puesto del ranking nacional (345.410), tras Madrid y Barcelona, y por encima de provincias de mayor entidad poblacional (población de derecho), como Valencia o Málaga, en la propia fachada mediterránea espańola, o de Sevilla, en la atlántica.

La mayoría de los municipios litorales y prelitorales de la provincia de Alicante han llevado a cabo el proceso de modernización de las funciones productivas apoyándose en lo que se ha denominado "turismo residencial". Este concepto se ha utilizado desde finales de los ańos setenta para explicar los cambios sociales, económicos y territoriales generados por el incremento de dos formas de movilidad humana, como son el turismo y la migración (Huete \& Mantecón, 2010). Los residentes de estas viviendas secundarias constituyen una amalgama social bajo la que se incluyen casuísticas diversas (Rodríguez, 2004; Huete, Mantecón \& Mazón, 2008). Según Moreno, Alcalde y Lurbe (2004), se diferencian cinco grupos de turistas o residentes según los motivos de su traslado: turistas extranjeros jubilados que vienen a España a pasar sus vacaciones y, por lo tanto, permanecen un corto período de tiempo; inmigrantes jubilados que pasan de turistas a residentes (grupo que coincide con personas que veraneaban en estas localidades antes de la jubilación y que deciden instalarse definitivamente en el municipio, buscando un lugar agradable donde pasar sus últimos años); jubilados o prejubilados que adquieren directamente una vivienda y trasladan a ella su residencia principal, atraídos por la bonanza climática; personas que emigraron antes de la jubilación por motivos varios (trabajo, matrimonios mixtos, etcétera), y que posteriormente regresan y se 
jubilan en España; y jubilados que emigran para ingresar en una residencia, de forma temporal o permanente. Son los tres primeros colectivos mencionados los que predominan en el litoral alicantino. Se trata de un proceso que ha caracterizado a amplios sectores del litoral mediterráneo español, entre los que cabe citar, por su dilatada tradición, las Islas Baleares (Salvà, 2002), la Costa del Sol (Raya \& Benítez, 2002; O’Reilly, 2005) o el litoral murciano (Monreal, 2001), al igual que el litoral mediterráneo europeo (Costa Azul francesa) o el de Florida (Estados Unidos) (Fernández \& Barrado, 2011). Nos encontramos, por tanto, ante una actividad que se asocia, por un lado, a las formas de veraneo tradicionales; y por otra, a nuevos estilos de vida y tipos de movilidad residencial vinculados a la construcción masiva de viviendas secundarias (Vera, 2005).

\section{Objetivos y metodología}

La actividad turística, y especialmente la turística residencial, ha producido un cambio en el modelo urbano y demográfico en los municipios de la fachada mediterránea. La provincia de Alicante es un claro ejemplo de ello. Estas nuevas formas de movilidad han sido integradas en un proceso de urbanización que se basa en la masiva producción de segundas viviendas en áreas con un potencial atractivo turístico. La plasmación territorial de estas dinámicas en las regiones mediterráneas españolas ha dado lugar a nuevos enclaves urbanos separados de los núcleos poblacionales tradicionales (Huete \& Mantecón, 2011), que han generado diversas repercusiones y conflictos territoriales, más si cabe cuando este fenómeno se ha producido en un corto período de tiempo (desde mediados de 1990), como ha acontecido en el sur de la provincia de Alicante, a excepción de Torrevieja (Vera, 1984).

El incremento de la población y de los espacios urbanizados ha sido causa de notables trasformaciones territoriales, muy evidentes desde el punto de vista de la articulación del territorio. No menos significativas resultan las sociales, asociadas a la llegada de importantes contingentes poblacionales. A tenor de las dinámicas descritas, los objetivos de este artículo son: i) poner de manifiesto las causas que explican la llegada de población procedente del centro y norte de Europa; ii) evidenciar las diversas fases de llegada de esta población, desde los puntos de vista tanto temporal como espacial, y su influencia en la expansión de los usos urbanoresidenciales; iii) evidenciar las repercusiones territoriales que conlleva el proceso de expansión residencial. Estas consecuencias se han analizado en una doble escala -provincial y de detalle-, a modo de estudio de caso. Concretamente, el área de estudio seleccionada está compuesta por nueve municipios del litoral y prelitoral de la provincia de Alicante, que corresponden a localidades donde el fenómeno del turismo residencial ha sido muy intenso; se distingue, a su vez, entre litoral sur (San Fulgencio, Rojales, Torrevieja y San Miguel de Salinas) y norte (Calpe, Altea, Polop, La Nucía y L'Alfas del Pi), dados los diferentes ritmos de implantación de las dinámicas turístico-residenciales (figura 1).

A nivel metodológico, el artículo adopta un enfoque integrado, por el cual se combinan las metodologías cuantitativas y cualitativas. Por lo que se refiere a las primeras, se centran en el análisis de la evolución registrada por la población 
y viviendas, realizado a partir de la información proporcionada por el Instituto Nacional de Estadística (INE) y el Ministerio de Fomento. Para la población total, se han consultado los datos a partir de 1960 para determinar su dinámica, mientras que para las cifras de extranjeros se han utilizado las disponibles a partir de 1991, dada su no desagregación en los censos anteriores. En las viviendas, el estudio se circunscribe a los censos 1991, 2001 y 2011; sus datos se complementan con los disponibles en el Ministerio de Fomento y con la restitución fotogramétrica de las fotografías aéreas correspondientes al vuelo de 1978 y 2012, orientada a determinar los cambios en los usos del suelo. La información estadística oficial se completa con la obtenida en el análisis y tratamiento estadístico de los datos obtenidos de 122 entrevistas que se llevaron a cabo en urbanizaciones de los nueve municipios del área de estudio. En ellas, a través de una serie de cuestiones, se trataba de profundizar en el conocimiento de los factores (ambientales, sociales, culturales, económicos) que determinaban la elección de este territorio como segunda y, en ocasiones, residencia principal, así como avanzar en los elementos que incidían en la elección de la tipología urbana.

\section{Figura 1 | Área de estudio (Provincia de Alicante, España)}

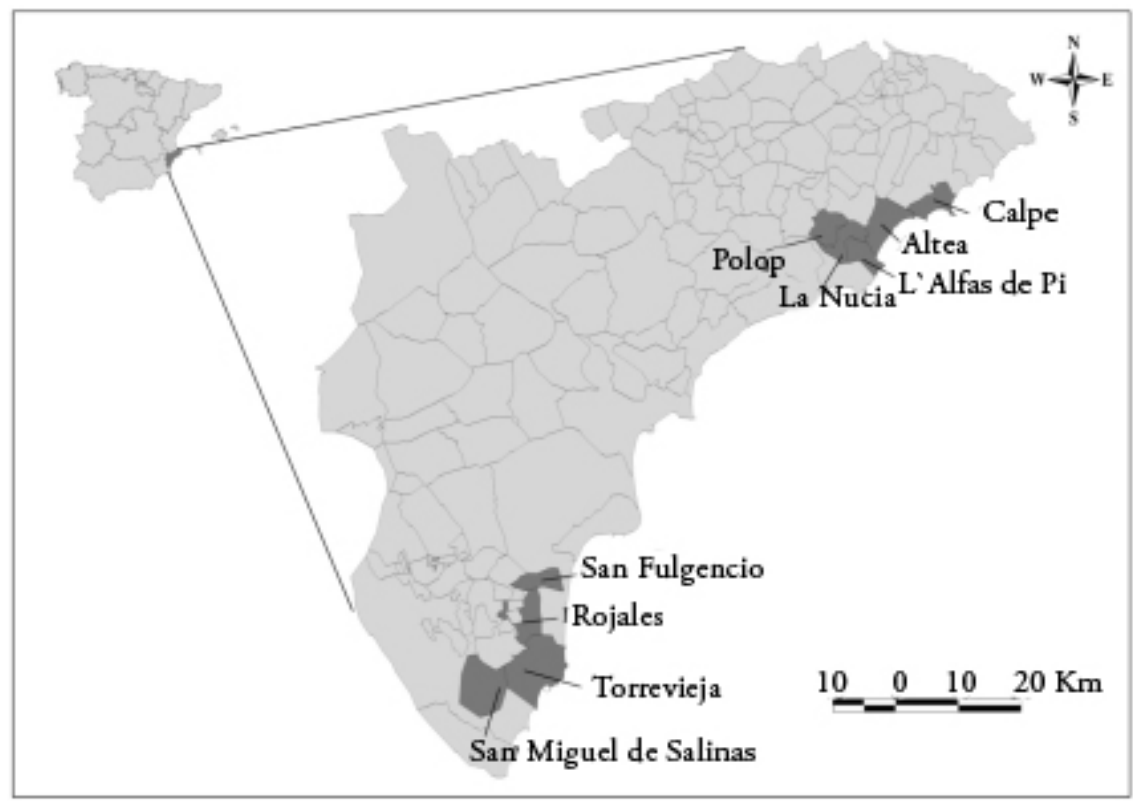

FUENTE ELABORACIÓN PROPIA

En esta publicación, concretamente, se van a examinar los resultados relacionados con los aspectos generales de los residentes de las urbanizaciones de estos municipios (nacionalidad, edad, situación laboral y nivel educativo) y de sus viviendas (régimen de propiedad, tipo de ocupación, habitantes por hogar y período de residencia en el área de estudio). Se trata, por tanto, de contrastar datos estadísticos oficiales con otros que 
nos proporcionan las fuentes señaladas, y que resultan fundamentales para explicar el atractivo de los municipios del litoral de Alicante y dar cuenta de los cambios sociales y territoriales acaecidos en ellos. La metodología que se utilizó para escoger la muestra de viviendas que serían encuestadas en las urbanizaciones objeto de análisis se compone de varias partes. La primera consistió en obtener fotografías áreas del área de estudio. La segunda fue la fotointerpretación o diferenciación de los espacios urbanos que se clasifican como urbanizaciones, eliminando aquellos que corresponden a espacios urbanos compactos. Obtenida la cartografía, la siguiente tarea fue seleccionar los municipios representativos de las diversas etapas de expansión urbano-residencial identificadas y la muestra aleatoria de las urbanizaciones y de los entrevistados. En el primero de los casos, se seleccionaron nueve municipios (cinco en el litoral norte y cuatro en el sur) y veintiocho urbanizaciones, siendo el número de urbanizaciones de cada municipio proporcional al número total de urbanizaciones existentes en él. Con respecto a las entrevistas, se obtuvo una ratio de éxito de un 20\%, es decir, para las 122 entrevistas realizadas entre mayo y julio de 2011 se llevaron a cabo unos 650 intentos.

\section{Dinámicas poblacionales positivas caracterizadas por la llegada de población extranjera}

El proceso turístico-residencial impone un incremento constante de la producción de oferta inmobiliaria e, igualmente, requiere la disponibilidad de una población en aumento, que se obtiene mediante un doble flujo inmigratorio de personas atraídas, de un lado, por las nuevas oportunidades laborales; y de otro, por las posibilidades de ocio existentes en un espacio en el que pueden disfrutar de un clima de filiación mediterránea con inviernos suaves, y precios asequibles (Mantecón, 2012).

CUADro 1 | Evolución de la población en la Comunidad Valenciana y en la provincia de Alicante (1960-2011)

\begin{tabular}{|l|c|c|c|c|c|c|}
\cline { 2 - 7 } \multicolumn{1}{c|}{} & \multicolumn{1}{c|}{$\mathbf{1 9 6 0}$} & I970 & I98 I & I99I & 200I & 20I I \\
\hline C. Valenciana & 2.479 .078 & 3.073 .255 & 3.646 .870 & 3.857 .234 & 4.162 .776 & 5.009 .931 \\
\hline Prov. Alicante & 711.413 & 920.105 & 1.149 .185 & 1.295 .563 & 1.461 .925 & 1.852 .166 \\
\hline
\end{tabular}

FUENTE INSTITUTO NACIONAL DE ESTADÍSTICA (INE). ELABORACIÓN PROPIA

En el período comprendido entre 2001 y 2011, la población en España, según el Censo de habitantes, aumentó en unos 6,09 millones, alcanzando a finales de 2011 los 46,8 millones (INe, 2012). La de la Comunidad Valenciana ha registrado una dinámica alcista desde la década de los ańos sesenta, tendencia que se inserta en un proceso de atracción de población por motivos laborales asociados al desarrollo industrial, al despegue turístico y a la reactivación de la agricultura comercial en un primer momento; y, posteriormente, a motivaciones turístico-residenciales. Entre 1960 y 2011, la población se ha multiplicado por 2 en la Comunidad Valenciana y por 2,6 en la provincia de Alicante (cuadro 1), cifras que contrastan con la dinámica nacional, donde lo ha hecho por 1,3, al pasar de 30,5 a 48,8 millones entre 1960 
y 2011, respectivamente. Esta corriente general, no obstante, encubre realidades diversas que, grosso modo, podemos sintetizar en un litoral que registra importantes incrementos poblacionales, frente a las áreas de interior, que experimentan notables pérdidas de población como consecuencia del éxodo rural (Piqueras, 2012).

CUADro 2 Evolución de la población en los municipios del área de estudio (1960-2011)

\begin{tabular}{|l|r|r|r|r|r|r|}
\cline { 2 - 7 } \multicolumn{1}{l|}{} & I960 & I970 & I98 I & I99I & 200I & 20I I \\
\hline Municipios litoral norte \\
\hline L'Alfas del Pi & 960 & 2.614 & 5.049 & 9.075 & 11.103 & 19.802 \\
\hline Altea & 5.620 & 8.804 & 11.154 & 12.286 & 15.910 & 22.385 \\
\hline Calpe & 2.177 & 3.399 & 8.032 & 10.683 & 18.881 & 23.241 \\
\hline La Nucía & 1.452 & 2.031 & 3.419 & 6.078 & 6.587 & 17.096 \\
\hline Polop & 1.402 & 1.574 & 1.766 & 1.855 & 2.300 & 4.159 \\
\hline Municipios litoral sur & & & & & \\
\hline Rojales & 4.470 & 4.006 & 4.117 & 5.277 & 8.489 & 17.986 \\
\hline San Fulgencio & 1.602 & 1.535 & 1.549 & 1.591 & 4.039 & 9.572 \\
\hline San Miguel de Salinas & 2.164 & 2.278 & 2.301 & 2.955 & 4.310 & 6.391 \\
\hline Torrevieja & 9.564 & 9.735 & 12.321 & 25.014 & 50.953 & 90.097 \\
\hline
\end{tabular}

FUENTE INSTITUTO NACiONAL DE ESTAdística (INE). ELABORACión PROPIA

Representativos de la dinámica que caracteriza a los municipios litorales y prelitorales valencianos desde 1960 hasta la actualidad son los nueve municipios analizados. Altea, por ejemplo, ha pasado de 5.620 habitantes en 1960, a 22.385 en 2011, es decir, multiplica por 4 su población en cincuenta años. Torrevieja, en el mismo período de tiempo, por 9,5, al pasar de 9.654 habitantes en 1960 a 90.097 en 2011. Estas cifras corresponden a población censada, a la cual hay que incorporar la flotante, es decir, la constituida por aquellos que residen en estos municipios una parte sustancial del año, pero que continúan manteniendo su residencia principal en su ciudad de procedencia, y los residentes y turistas que únicamente habitan en ella en el período estival. En 1960, excepto Torrevieja -que se iba configurando como la ciudad más importante del litoral sur de la provincia gracias a la industria salinera, la pesca y el turismo-, las demás eran poblaciones pequeńas cuya economía se basaba principalmente en la agricultura. El notable incremento poblacional que todos esos municipios registran a partir de la década de los sesenta es resultado de la irrupción de las nuevas funciones económicas.

El incremento poblacional registrado, sin embargo, no es homogéneo ni en el tiempo ni en el espacio. El análisis de la evolución demográfica de la población pone de manifiesto la existencia de tres etapas asociadas a la implantación y posterior difusión de las actividades turístico-residenciales (cuadro 2). La primera, que es la más dilatada, se produce a partir de las décadas de 1960 y 1970, y afecta únicamente a municipios litorales. La población de las localidades de la primera línea de 
costa tanto del litoral norte (Calpe, Altea y L'Alfas del Pi) como del sur (Torrevieja) creció notablemente, ligada al despegue de las actividades turísticas (Vera, 1984). Tal tendencia se prolonga en el tiempo hasta la actualidad, siendo este incremento muy elevado en el último intercensal, con porcentajes similares a los de la etapa inicial. Altea, por ejemplo, vio cómo su población se ampliaba en 3.184 habitantes entre 1960 y 1970, lo que suponía un aumento de casi un 40\%. Este se debió en un primer momento a la llegada de población nacional (de regiones como Castilla-La Mancha, Andalucía o Murcia, y de población de localidades cercanas y del interior de Alicante) para trabajar en el sector turístico y en el de la construcción. A esta población hay que sumar la venida gradual de residentes extranjeros procedentes del norte y centro de Europa, que compraron una casa en las urbanizaciones del litoral alicantino, atraídos por la bonanza climática, suelo y viviendas más baratas y un coste de vida notablemente inferior al de sus países de procedencia. Además de su marcada localización costera, un segundo rasgo que caracteriza a esta primera etapa es la diferente intensidad del proceso entre el litoral norte y el sur. En la década de los años sesenta y setenta, las actividades turísticas se concentraron básicamente en el sector septentrional. Factores como el desarrollo turístico de Benidorm, la mejora de la accesibilidad (con la inauguración del aeropuerto de Alicante-Elche y la construcción de la autopista Ap-7), una mayor calidad paisajística (colinas y espacios forestales intercalados entre áreas cultivadas), la fragmentación parcelaria y una reducida rentabilidad agraria que favorecía la venta de parcelas agrarias y su adquisición por particulares, entre otros, explican el inicio y posterior expansión de esta actividad. En el sur, el turismo se centralizó a partir de 1970 mayoritariamente en Torrevieja, coincidiendo con el gran desarrollo urbano-turístico que supuso la consolidación de este municipio como el principal destino vacacional valenciano (Bańos, 1999), y como aquel donde el segmento de población nacional que adquiría una segunda residencia era mayoritario en esta etapa inicial. Su población registra un incremento imperceptible entre 1960 y 1971 (171 habitantes), para aumentar notablemente a partir de ese momento (un 20\% en el intercensal 1971-1981), porcentaje que se eleva de manera espectacular en el último intercensal, cuando se sitúa en torno al $44 \%$.

Las dinámicas señaladas no solo afectaron a los municipios de primera línea de costa (Altea, Calpe, L'Alfas del Pi o Torrevieja), sino que progresivamente fueron influyendo en municipios prelitorales, en un primer momento los del litoral norte (a partir de 1980) y más recientemente (a partir de 1990) en el litoral sur. En ambas fases, las causas de este incremento poblacional se asocian con la saturación del litoral y la búsqueda de áreas próximas a la costa con mayor calidad paisajística y precios del suelo más bajos. Es el caso de La Nucía, su crecimiento se asocia a la saturación del suelo urbano en municipios próximos (L’Alfas del Pi y Benidorm). Frente a la atonía entre 1960 y 1971, en el intercensal 1981-1991 su población se duplicó, al pasar de 3.419 habitantes a más de 6.000, incremento que se mantendría sostenido en el tiempo hasta la última década, en que vuelve a aumentar notablemente, cuando pasa de 6.078 en 1991 a 17.096 en 2011; es decir, en tan solo dos décadas la población se ha multiplicado por tres.

A partir de la década de los noventa, el proceso de crecimiento poblacional afecta a municipios de segunda línea de costa en el litoral sur, que hasta ese momento se 
caracterizaban por unas dinámicas demográficas estancadas y una base económica fundamentada en la agricultura. En esta segunda línea de costa del litoral sur se encuentran localidades como Rojales y San Fulgencio, que representan el mismo esquema implantado en todo el prelitoral de este sector (Daya Nueva, Daya Vieja, Formentera del Segura, Benijófar, Algorfa, Los Montesinos, San Miguel de Salinas, etcétera). Ambos municipios vieron duplicada su población entre 1991 y 2011. El primero, por ejemplo, ha pasado de 8.489 a 17.986 habitantes. Esta intensa dinámica poblacional se vincula a factores ya comentados, como es la saturación urbana de la costa y la difusión hacia áreas interiores; pero también a otros nuevos: su elevada intensidad se ve favorecida por un desarrollo en las comunicaciones aéreas (creación de una amplia oferta de vuelos de bajo coste) y terrestres (mejora de numerosas vías de comunicación gracias a los fondos de desarrollo regional procedentes de la Unión Europea), que van a permitir conectar áreas emisoras y receptoras, así como por la configuración de una enorme oferta de viviendas a precios mucho más bajos que los de los países de procedencia o de otros sectores litorales, tanto nacionales como provinciales (litoral norte).

A partir de mediados de la década de 1990, el incremento de la población se asocia a la llegada de población procedente de otras regiones espańolas, pero sobre todo de importantes contingentes poblacionales del centro y norte de Europa. Según el Censo 2001, a la fecha el porcentaje de población extranjera ascendía al 4,5\% del total nacional, elevándose al 12,1\% en 2011 (INE, 2012), cifra que adquiere una mayor magnitud en la escala regional y local. En las provincias ribereñas del Mediterráneo, el colectivo integrado por población oriunda del centro y norte de Europa se sitúa, salvo alguna excepción, en proporciones superiores al 15\% del total poblacional, especialmente en aquellos lugares donde el desarrollo residencial se remonta a la década de los ańos setenta del siglo xx (Baleares, Málaga y Alicante). Mayor relevancia adquieren las cifras si se analiza la población venida del centro y norte de Europa en relación con la población extranjera total (figura 2), frente a la cual se sitúa, salvo excepciones, por encima del $50 \%$.

FIGURA 2 Porcentaje de europeos (excluida la población española) sobre el total de extranjeros (2001-2011)

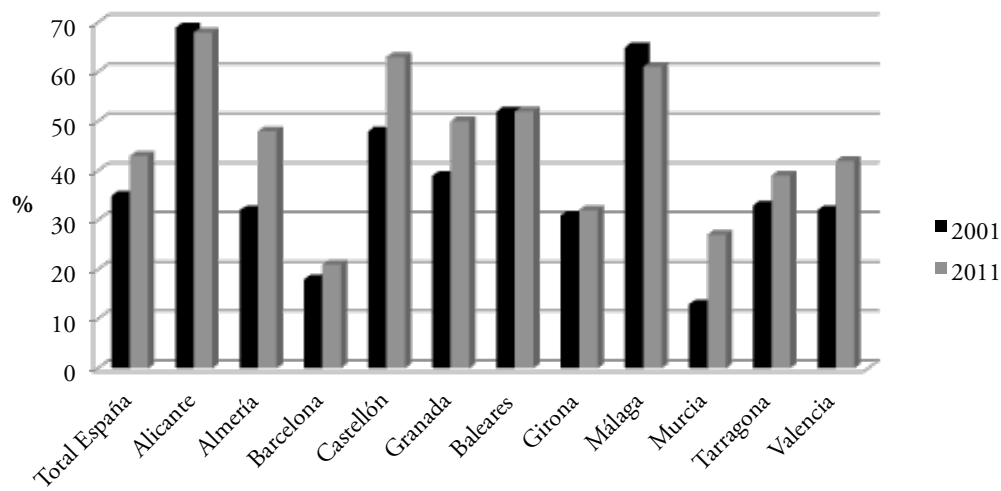

FUENTE INSTITUTO NACIONAL DE ESTADÍSTICA (INE). ELABORACIÓN PROPIA 
CUADRo 3 | Evolución de la población extranjera (1991-2011)

\begin{tabular}{|c|c|c|c|c|c|c|}
\hline & \multicolumn{2}{|c|}{ I99I } & \multicolumn{2}{|c|}{$200 I$} & \multicolumn{2}{|c|}{$201 \mathrm{I}$} \\
\hline & $\begin{array}{c}\text { POBLACIÓN } \\
\text { EXTRANJERA }\end{array}$ & $\begin{array}{c}\% \text { РОВ. } \\
\text { EXTRANJERA }\end{array}$ & $\begin{array}{c}\text { POBLACIÓN } \\
\text { EXTRANJERA }\end{array}$ & $\begin{array}{c}\text { \% РOB. } \\
\text { EXTRANJERA }\end{array}$ & $\begin{array}{c}\text { POBLACIÓN } \\
\text { EXTRANJERA }\end{array}$ & $\begin{array}{c}\text { \% РОВ. } \\
\text { EXTRANJERA }\end{array}$ \\
\hline C. Valenciana & 54.213 & 1,40 & 215.673 & 5,18 & 756.772 & 15,11 \\
\hline $\begin{array}{l}\text { Provincia de } \\
\text { Alicante }\end{array}$ & 12.916 & 1,00 & 125.531 & 8,59 & 380.098 & 20,52 \\
\hline \multicolumn{7}{|c|}{ Municipios litoral norte } \\
\hline L'Alfas del Pi & 4.927 & 54,29 & 4.004 & 36,06 & 10.427 & 52,66 \\
\hline Altea & 900 & 7,33 & 2.497 & 15,69 & 7.627 & 34,07 \\
\hline Calpe & 3.421 & 32,02 & 9.139 & 48,40 & 12.149 & 52,27 \\
\hline La Nucía & 3.575 & 58,82 & 2.113 & 32,08 & 6.045 & 35,36 \\
\hline Polop & 115 & 6,20 & 454 & 19,74 & 1.375 & 33,06 \\
\hline \multicolumn{7}{|c|}{ Municipios litoral sur } \\
\hline Rojales & 990 & 18,76 & 3.901 & 45,95 & 12.868 & 71,54 \\
\hline San Fulgencio & 143 & 8,99 & 1.704 & 42,19 & 6.794 & 70,98 \\
\hline $\begin{array}{l}\text { San Miguel de } \\
\text { Salinas }\end{array}$ & 315 & 10,66 & 1.483 & 34,41 & 3.512 & 54,95 \\
\hline Torrevieja & 4.590 & 18,35 & 14.154 & 27,78 & 41.233 & 45,77 \\
\hline
\end{tabular}

FUENTE INSTITUTO NACIONAL DE ESTADíSTICA (INE). ELABORACióN PROPIA

Este proceso se ha acentuado de manera muy notable en el último intercensal. En la Comunidad Valenciana, de los 847.155 nuevos habitantes censados en 2011 con respecto a 2001, el 64\% eran extranjeros, dinámica que se repite en el caso de la provincia de Alicante. El análisis en la escala local refleja la intensidad de la llegada de población europea en la última década. Llamativos resultan municipios como Rojales y San Fulgencio (sur de la provincia de Alicante), en los que el porcentaje de extranjeros ha pasado del 35\% al 70\% del total poblacional entre 2001 y 2011, respectivamente. Rojales y San Fulgencio ocupan los primeros puestos del ranking de municipios españoles con mayor número de extranjeros (cuadro 3), correspondiendo a ellos prácticamente la totalidad del incremento demográfico, frente a la atonía de la población española. Estos datos corresponden a cifras oficiales, es decir, a población censada. A ella habría que incorporar aquellos habitantes que, por motivos diversos, mantienen su residencia principal en sus países de procedencia, aunque residen en estas tierras la mayor parte del año. A pesar de las diversas campañas llevadas a cabo por diferentes municipios, el porcentaje de residentes sigue siendo significativo, con cifras que se sitúan entre el $25 \%$ y el $30 \%$, según municipios. El análisis de preguntas como "régimen de propiedad" y "período de residencia en el área de estudio" reflejan la importancia del fenómeno. El predominio de la vivienda en propiedad es abrumador en el área de estudio ( $90 \%$ de los entrevistados). De ese porcentaje de viviendas en propiedad, el 66,12\% comprende viviendas principales, mientras que el resto $(33,88 \%)$ se trata de segundas residencias. Interesante resulta 
también la información derivada de quienes las utilizan como segunda residencia. La respuesta mayoritaria, con un 51\%, es que las habitan durante el verano, pero hay también población que lo hace durante otros períodos del año, como puede ser de enero a mayo (el $24,4 \%$ de las respuestas), o de octubre a diciembre (el $8,8 \%$ ), o durante más de medio ańo (13,3\%). Las cifras evidencian que la segunda residencia se asocia con uso estival, pero su utilización durante el resto de los meses tiene una clara relación con población no espańola, como refleja la combinación entre este ítem y el de nacionalidad.

El incremento de la población extranjera es paralelo a las dinámicas turísticoresidenciales, es decir, a los procesos de difusión de los usos urbanos en el territorio. Atendiendo a estos, se pueden diferenciar tres etapas desde el punto de vista cronológico. La primera, la más antigua, interesaría a los municipios estrictamente litorales y, sobre todo, al litoral norte, donde los porcentajes de este colectivo superaba el $30 \%$, frente a su ínfimo valor (alrededor de un 1\%) a escala tanto regional como provincial. Calpe, por ejemplo, en 1991 tenía censados 3.421 extranjeros, cifra que se incrementó hasta los 12.149 en 2011, es decir, en tan solo veinte años, los extranjeros en esta localidad se cuadriplicaron, para llegar a representar aproximadamente la mitad de la población total (23.241 habitantes). Una dinámica incluso más intensa registra el municipio de L'Alfas del Pi, cuya población extranjera en 1991 superaba ya el $50 \%$. Torrevieja, en el litoral sur, presentaba porcentajes notablemente inferiores. Ello es debido a que en esta primera etapa de turismo residencial atrajo sobre todo a turistas españoles, que adquirieron su segunda residencia en este municipio.

Este proceso se propagará hacia municipios prelitorales y, sobre todo, en el litoral sur, a partir del intercensal 1991-2001, siendo su rasgo más significativo el de su intensidad en la última década, que supera notablemente a la registrada en las anteriores. Representativos resultan los municipios de la denominada segunda línea de costa. Polop, por ejemplo, en 1991 únicamente tenía censados como extranjeros a 115 personas, es decir, el 6,2\% del total de la población, frente a los 1.375 de 2011, que corresponden al 36\% del total poblacional. Es, sin embargo, el litoral sur donde la llegada de población extranjera ha sido más espectacular, atendiendo a su intensidad y al breve período de tiempo en que se ha producido. En poco más de una década, la población extranjera ha pasado de representar menos del 20\%, a situarse en valores próximos al 70\%. Rojales, por ejemplo, contaba en 1991 con tan solo 990 extranjeros, cifra que se eleva a 3.901 en 2001 y a 12.868 en 2011, que representan el $70 \%$ del total de la población. Dinámica similar se observa en San Fulgencio, donde la población extranjera pasó de representar el 8\% en 1991 al 71\% en 2011. La creación de una amplia oferta de viviendas a precios muy asequibles, la amplia oferta de vuelos de bajo coste y las numerosas campañas de captación de clientes en las áreas de procedencia por las promotoras urbanas, justifican la intensidad del proceso.

El ámbito de procedencia de esta población inmigrante proporciona información también relevante sobre su tipología. De manera general, en la provincia de Alicante, el porcentaje de extranjeros europeos se situaba próximo al $70 \%$ en 2011 (figura 2). Se trataba de un colectivo constituido por jubilados o prejubilados procedentes del centro y norte de Europa (mayoritariamente ingleses y alemanes, 
y recientemente colectivos del este de Europa, sobre todo en el litoral sur), que han comprado una vivienda en esta franja costera atraídos por la benignidad de las condiciones climáticas, los servicios disponibles, la existencia de viviendas a precios más baratos que los de su área de procedencia, la agilización de los trámites para el desarrollo de actuaciones urbanísticas asociadas a la aprobación de la Ley 6/1994, de 15 de noviembre, de la Generalidad Valenciana, Reguladora de la Actividad Urbanística (LRAU), o la existencia de importantes colectivos de estas nacionalidades, que facilitan las relaciones sociales. Estos rasgos se acentúan en la escala local, como corrobora el análisis de las variables "edad" y "nacionalidad" en las entrevistas realizadas. Con este análisis se evidencia que nos encontramos en urbanizaciones donde predomina la población de más de 60 años (el 65\% de los entrevistados supera esta edad). Este dato resulta más ilustrativo si se interrelacionan ambos ítems, edad y nacionalidad. El 74\% de los extranjeros supera los 60 ańos, frente a los españoles, que es el único colectivo con población de menos de 60: concretamente, el $61 \%$ tiene entre 25 y 59 ańos. Nos encontramos, por tanto, ante dos realidades contrastadas: una mayoritaria, que corresponde a población jubilada o prejubilada que, tras finalizar su edad laboral, decide trasladar su residencia a ámbitos más benignos desde una óptica climática; y una minoritaria, caracterizada por población adulta joven que adquiere una vivienda fuera del núcleo urbano consolidado, generalmente buscando una mejor calidad de vida asociada a espacios verdes, menor congestión urbana e, incluso, precios más bajos. Los resultados del ítem "situación laboral" corroboran estas afirmaciones: el 55,47\% de los entrevistados son jubilados, el $26,78 \%$ trabaja, el $14,04 \%$ estudia y el $3,71 \%$ se encuentra en paro, lo que reitera la dualidad entre extranjeros y espańoles. Los primeros corresponden al primer valor, los otros tres resultados se asocian, mayoritariamente, con la cohorte de población nacional.

FIGURA 3 | Porcentaje de población extranjera europea, no europea y española (2011)

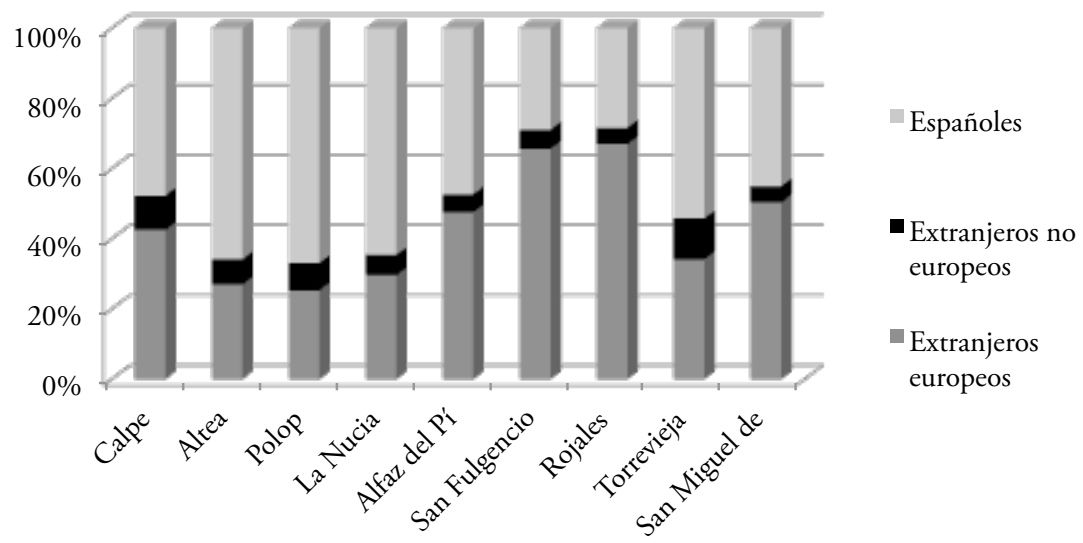

FUENTE INSTITUTO NACIONAL DE ESTADÍSTICA (INE). ELABORACIÓN PROPIA 
El análisis de la figura 3 refleja una cierta heterogeneidad entre los municipios del área de estudio, como resultado de la mayor o menor implantación del turismo residencial tanto en el tiempo como en el espacio, y de la existencia de una mayor o menor diversificación productiva. Rasgo común a todos ellos es el poco relevante peso de la emigración no europea, lo que da cuenta de que la atracción por motivos laborales es insignificante, a diferencia de otros ámbitos regionales, como pueden ser las capitales provinciales. En el litoral norte, por ejemplo, la localidad de Calpe tenía censados 12.149 extranjeros en 2011, de los que el $81 \%$ eran europeos, con predominio de las nacionalidades inglesa (4.334) y alemana (3.821). Valores similares da el Censo para L'Alfas del Pi, Altea, Polop o La Nucía. Porcentajes superiores, en cambio, se alcanzaban en los municipios del prelitoral sur, donde el colectivo británico era mayoritario a la fecha. En Rojales, el 93\% del total de extranjeros censados (12.057) eran europeos, predominando de manera notable el colectivo británico, que ascendía a 9.181 habitantes.

\section{Repercusiones territoriales}

Un factor explicativo de la fuerte expansión de las funciones residenciales en el litoral mediterráneo es la adquisición de viviendas por población procedente de otras regiones espańolas; pero, sobre todo, por la fuerte demanda por parte de inmigrantes europeos, sin olvidar el carácter especulativo asociado a la obtención de ingentes beneficios por la construcción de segundas residencias en suelos rústicos. Propietarios de tierras, agricultores y profesiones liberales y, sobre todo, grandes inversores nacionales y extranjeros, adquieren parcelas como forma de invertir en un negocio que hasta el estallido de la burbuja inmobiliaria en 2008 ofrecía un alto beneficio, asociado a la recalificación de suelos rústicos para la construcción de segundas residencia a partir de su parcelación.

El incremento poblacional y la llegada de colectivos procedente del centro y norte de Europa han generado notables repercusiones territoriales, que podemos sintetizar en un aumento importante del parque de viviendas, que entre 1991 y 2011 se ha incrementado en un 33\%. Tal porcentaje se amplía significativamente en la escala municipal, donde los aumentos, salvo excepciones, superan el 50\%, e incluso en casos excepcionales llega a valores próximos al 70\%, como sucede en el municipio de Rojales (cuadro 4).

El desarrollo de las superficies urbanizadas ha ido en consonancia con el incremento del parque de viviendas. En el área de estudio, la superficie urbanizada ha pasado de unos 12,5 millones de metros cuadrados en 1978 a más de 55,7 en 2012 (cuadro 5).

La expansión de los usos residenciales se caracteriza por una serie de rasgos que definen aquel modelo urbano que comenzó a difundirse desde 1960, y que se traducen en significativas repercusiones sociales y territoriales. En primer lugar, esta expansión se lleva a cabo en espacios donde predominaban los usos agrícolas e, incluso en ocasiones, sobre sectores de monte bajo y matorral. El cambio de paisaje es notable, ya que chalés y adosados aparecen, bien de manera dispersa por gran parte de los términos municipales, bien configurando macrourbanizaciones como 
consecuencia del cambio de la tipología del suelo (de no urbanizable a urbanizable), pero también por la venta de parcelas rústicas cuyos propietarios veían esta transacción como un negocio, y en las que posteriormente se construía una vivienda.

CUADRo 4 | Evolución del parque de viviendas (1991-2011)

\begin{tabular}{|l|r|r|r|c|}
\cline { 2 - 5 } \multicolumn{1}{l|}{} & \multicolumn{1}{c|}{ I99I } & 200I & 20I I & $\begin{array}{c}\text { \% DE INCREMENTO } \\
\text { DEL PARQUE DE } \\
\text { VIVIENDAS }\end{array}$ \\
\hline Total C. Valenciana & 2.094 .033 & 2.547 .775 & 3.147 .678 & 33,47 \\
\hline Provincia de Alicante & 789.481 & 1.004 .188 & 1.274 .325 & 38,04 \\
\hline Provincia de Castellón & 269.277 & 325.689 & 420.516 & 35,96 \\
\hline Provincia de Valencia & 1.035 .275 & 1.217 .898 & 1.452 .838 & 28,74 \\
\hline Municipios del área de estudio & \multicolumn{3}{|c|}{} \\
\hline Alfas del Pi & 6.555 & 11.017 & 12.417 & 47,20 \\
\hline Altea & 9.600 & 12.397 & 16.437 & 41,59 \\
\hline Calpe / Calp & 16.317 & 18.385 & 24.957 & 34,61 \\
\hline La Nucía & 4.739 & 6.094 & 8.774 & 45,98 \\
\hline Polop & 1.184 & 1.683 & 2.786 & 57,50 \\
\hline Rojales & 3.726 & 7.105 & 14.263 & 73,87 \\
\hline San Fulgencio & 6.401 & 6.132 & 8.661 & 26,09 \\
\hline San Miguel de Salinas & 2.620 & 4.872 & 5.966 & 56,08 \\
\hline Torrevieja & 53.251 & 1.02 .355 & 122.338 & 56,47 \\
\hline
\end{tabular}

FUENTE INSTITUTO NACIONAL DE ESTADíSTICA (INE). ELABORACIÓN PROPIA

Figura 4 Municipio de San Fulgencio (núcleo urbano en la parte inferior izquierda y las urbanizaciones en la parte superior derecha)

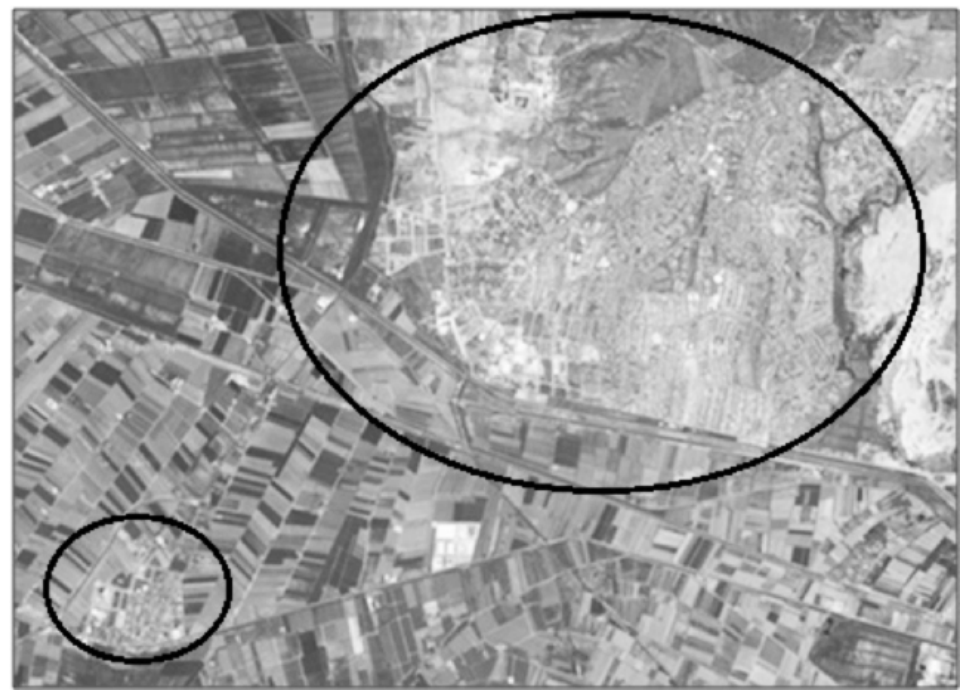

FUENTE HTTPS://MAPS.GOOGLE.ES/MAPS. ELABORACIÓN PROPIA 
En segundo lugar, estos nuevos espacios urbanos se instalan al margen de los núcleos urbanos tradicionales, configurando nuevas áreas residenciales que, generalmente, superan notablemente en tamańo a aquellos (figura 4). Es el caso, por ejemplo, de la localidad de San Fulgencio, donde las urbanizaciones se concentran en un núcleo consolidado de chalés y adosados conocido como la Marina-Oasis, situado a 3 kilómetros al noreste del núcleo urbano. Proceso similar se observa en el municipio de Rojales, donde se han construido macrourbanizaciones como Benimar, Pueblo Lucero, Ciudad Quesada o Dońa Pepa. No solamente se produce una segregación física, sino también social. El tratamiento estadístico de las entrevistas refleja que la mayoría de la población de estas urbanizaciones (el 75\%) está compuesta por residentes extranjeros procedentes del centro y norte de Europa.

CUADro 5 | Evolución de las superficies urbanizadas (1978-2012) en el área de estudio $\left(\mathrm{m}^{2}\right)$

\begin{tabular}{|c|c|c|c|c|}
\hline & & & & \\
\hline & & I978 & 2012 & $\begin{array}{c}\text { \% INCREMENTO SUP. } \\
\text { URBANIZADA }\end{array}$ \\
\hline \multirow{2}{*}{ L'Alfass del Pi } & núcleo urbano & 143.950 & 293.687 & 104,02 \\
\hline & urbanizaciones & 1.955 .104 & 5.668 .828 & 189,95 \\
\hline \multirow{2}{*}{ Altea } & núcleo urbano & 414.031 & 868.510 & 109,77 \\
\hline & urbanizaciones & 2.011 .952 & 8.518 .323 & 323,39 \\
\hline \multirow{2}{*}{ Calpe } & núcleo urbano & 209.571 & 447.945 & 113,74 \\
\hline & urbanizaciones & 2.792 .588 & 8.286 .012 & 196,71 \\
\hline \multirow{2}{*}{ La Nucía } & núcleo urbano & 137.390 & 350.310 & 154,97 \\
\hline & urbanizaciones & 1.067 .591 & 4.934 .951 & 362,25 \\
\hline \multirow{2}{*}{ Polop } & núcleo urbano & 128.749 & 191.782 & 48,96 \\
\hline & urbanizaciones & 105.648 & 1.182 .257 & $1.019,05$ \\
\hline \multirow{2}{*}{ Rojales } & núcleo urbano & 329.278 & 484.870 & 47,25 \\
\hline & urbanizaciones & 47.044 & 5.127 .387 & $10.799,13$ \\
\hline \multirow{2}{*}{ San Fulgencio } & núcleo urbano & 135.734 & 234.285 & 72,61 \\
\hline & urbanizaciones & 105.874 & 3.429 .868 & $3.139,58$ \\
\hline \multirow{2}{*}{ San Miguel de Salinas } & núcleo urbano & 217.418 & 514.361 & 136,58 \\
\hline & urbanizaciones & 0 & 1.570 .731 & $\infty$ \\
\hline \multirow{2}{*}{ Torrevieja } & núcleo urbano & 1.559 .734 & 2.465 .209 & 58,05 \\
\hline & urbanizaciones & 1.126 .900 & 11.164 .520 & 890,73 \\
\hline \multirow{3}{*}{ Total } & núcleo urbano & 3.275 .855 & 5.850 .959 & 78,61 \\
\hline & urbanizaciones & 9.430 .119 & 48.826 .507 & 417,77 \\
\hline & total & 12.488 .556 & 55.733 .836 & 346,28 \\
\hline
\end{tabular}

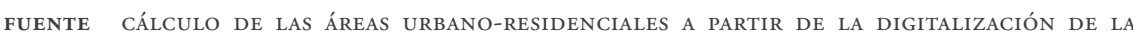
FOTOGRAFÍA AÉREA DEL EJÉRCITO DEL AIRE DE I 978 Y LAS ORTOFOTOS DEL PLAN NACIONAL DE ORTOGRAFÍA AÉREA (PNOA). ELABORACIÓN PROPIA 
Un tercer rasgo de estos espacios urbanos es un notable incremento de las superficies urbanizadas (cuadro 5). Esta ampliación va asociada, asimismo, a la difusión de un modelo urbano extensivo o semiextensivo, que contrasta con las tipologías urbanas más compactas y mayoritarias en estos territorios, hasta la implantación de los usos urbano-residenciales. Frente al sector concentrado y en altura caracterizado por construcciones en bloque, la expansión residencial se ha basado mayoritariamente en la difusión de nuevos modelos urbano-residenciales de baja densidad (unifamiliares con piscina y jardín), frente a los de media densidad (adosados) y a las edificaciones en altura (apartamentos), integrados ambos, mayoritariamente, en urbanizaciones privadas con jardín y piscina, que contrastan con los núcleos urbanos tradicionales (concentrados). Este proceso, por tanto, encubre una realidad dual (aumentos muy elevados en la tipología de urbanizaciones frente a otros más modestos en la de núcleo urbano), pero también intensidades diversas entre municipios. Los núcleos urbanos tradicionales, por ejemplo, se han incrementado en un $78 \%$, frente al $417 \%$ de las urbanizaciones.

El municipio de Rojales resulta representativo del proceso descrito. En los 35 años trascurridos desde 1978, el suelo ocupado por urbanizaciones ha pasado de unos 50.000 metros cuadrados a más de 5 millones; en cambio, el núcleo urbano únicamente se ha incrementado en unos 155.000 metros cuadrados. Continuando con el ejemplo de Rojales, en 1978 el porcentaje que representaba el suelo del núcleo urbano era el $87,4 \%$ del total, mientras que el 12,6\% correspondía a urbanizaciones de media y baja densidad (chalés y adosados). En 2012, esta distribución se ha invertido: el suelo del núcleo urbano ha descendido al 8,7\%, frente al $91,3 \%$ de las urbanizaciones de media y baja densidad (figura 5).

En cuarto lugar, se ha producido una difusión de los procesos de urbanización desde el litoral hacia sectores prelitorales. El inicio de la construcción de áreas residenciales en la franja costera se remonta a los ańos sesenta, con la llegada de población extranjera y nacional que adquiere una segunda residencia. La presión urbanística que el litoral experimentó durante los años sesenta, setenta y principios de los ochenta, provocó su saturación. La inexistencia de suelo disponible o sus altos precios en la primera línea de costa dio lugar a que los promotores inmobiliarios, en connivencia con las autoridades políticas municipales, favorecieran su difusión hacia sectores prelitorales, aprovechando en muchas ocasiones carreteras comarcales y viales locales para su propagación. Se repetían, de este modo, las dinámicas de producción urbana en las áreas mediterráneas prelitorales, y los modelos urbano-residenciales. El intenso proceso urbanizador acaecido desde mediados de los noventa y hasta el estallido de la burbuja inmobiliaria en 2008 se tradujo en una penetración hacia sectores más interiores, que imitaban, a su vez, la dinámica acontecida en los sectores litorales, configurándose, según áreas, una o dos coronas de urbanización desde la costa. En esta segunda línea de costa, por ejemplo, en el litoral sur se encuentran localidades como Rojales y San Fulgencio, que representan el mismo esquema implantado en todo el prelitoral a partir del núcleo primigenio de Torrevieja. En ellos han proliferado las urbanizaciones, principalmente de adosados y chalés pareados, que se extienden por todo el término municipal hasta formar macrourbanizaciones que superan en extensión al núcleo urbano tradicional. El 
proceso descrito se ha plasmado, desde el punto de vista territorial, en un continuo urbano, donde a menudo los viejos y los nuevos núcleos acaban por unirse.

FIGURA 5 | Evolución de la superficie urbano-residencial de Rojales

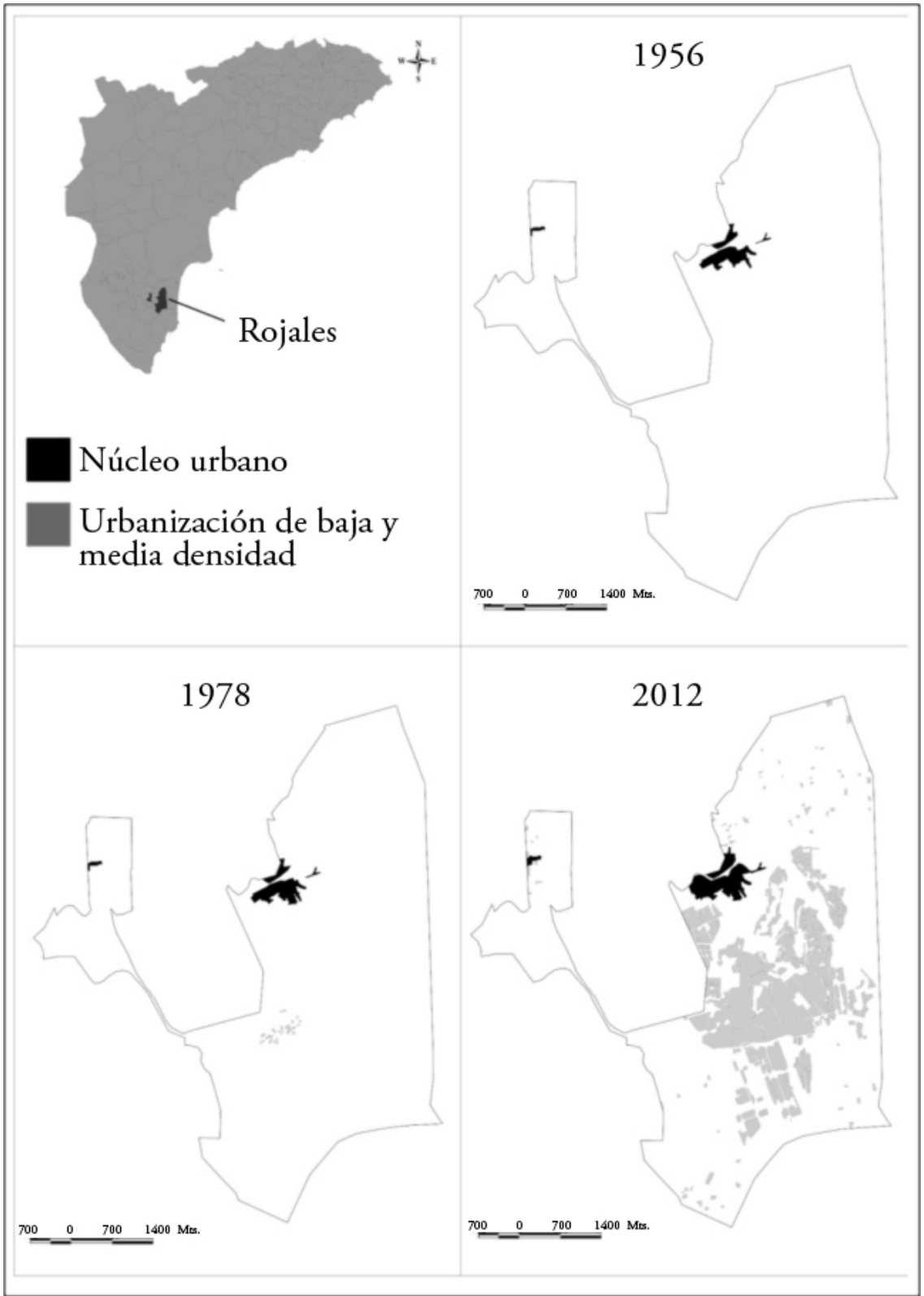

FUENTE ELABORACIÓN PROPIA A PARTIR DE LA DIGITALIZACIÓN DE LAS FOTOGRAFÍAS AÉREAS DEL VUELO AMERICANO DE I956, EJÉrCito DEL AIRE DE I978 y LAS ORTOFOTOS DEL PLAN NACIONAL DE ORTOGRAFÍA AÉREA (PNOA) 
En quinto lugar, es necesario considerar la intensidad del proceso. La difusión de los usos residenciales se desarrolla a partir de los años sesenta, en un primer momento en la franja litoral y posteriormente hacia la denominada segunda línea de costa. Este proceso general se acentúa significativamente a partir de mediados de la década de los noventa y hasta 2008, coincidiendo con un incremento notable en la llegada de población extranjera y el carácter especulativo del cambio en la clasificación y calificación de los usos del suelo. El análisis de la pregunta "tiempo que llevan viviendo los entrevistados en la vivienda" pone de manifiesto el período en el que se produjo su llegada a estas tierras, así como su evolución en el tiempo y la relación que se establece entre expansión residencial y llegada de población extranjera. Los que han residido en la vivienda 21 años o más (el 7,5\%) corresponden a los primeros extranjeros que establecieron su residencia en el lugar a finales de los años sesenta. Ese porcentaje se incrementa ligeramente en las dos siguientes cohortes (entre $10 \mathrm{y}$ 14 años y entre 15 y 20, respectivamente), que corresponden a la llegada de turistas en la década de los setenta, ochenta y principios de los noventa, acumulando entre ambas al 25\% de los residentes. El valor mayoritario, y que destaca sobre el resto, es el que corresponde a los que llevan viviendo entre 5 y 9 ańos (el 40\%). Esta situación coincide con el inicio del siglo XXI y con el máximo apogeo del boom inmobiliario. En los datos más recientes, se observa una disminución notable de los entrevistados que llevan viviendo menos de 4 años (coincidiendo con el estallido de la burbuja inmobiliaria y con la crisis económica).

El análisis desagregado del ítem "tiempo que llevan viviendo los entrevistados en la vivienda" entre las urbanizaciones del litoral norte y del sur pone de manifiesto diferencias significativas en los períodos de difusión de los usos urbano-residenciales entre estas dos áreas. Tales diferencias se relacionan con las fases de implantación y difusión de las actividades turístico-residenciales y su intensidad. En el litoral norte, los entrevistados que llevan viviendo entre 10 y 21 ańos representan al 36,6\% de la población, frente al $15 \%$ del litoral sur. Mayor contraste se produce en la cohorte "de 5 a 9 ańos". En el meridional, estos residentes representan el 46,60\%, frente al $33,3 \%$ del septentrional. Tales cifras vienen a justificar que el turismo residencial se asentó en un primer momento en el litoral norte y ha ido propagándose progresivamente hasta el estallido de la burbuja inmobiliaria. Se confirma también que, en el litoral sur, la llegada de población extranjera es más reciente, y además su ritmo ha sido más intenso en el tiempo, incrementándose notablemente a partir de mediados de los noventa, en coincidencia con el inicio del boom inmobiliario. También cabe destacar que los datos relativos al litoral sur, sobre todo los correspondientes a los períodos de una ocupación más dilatada en el tiempo (más de 15 años), se encuentran condicionados por las entrevistas realizadas en Torrevieja, donde su implantación fue más temprana. La combinación de la variable "municipio de residencia" y "años que habitan en la vivienda" evidencia que aquellos que residen más de quince años se localizan prácticamente en su totalidad en Torrevieja. Su presencia en el resto de municipios del litoral sur (segunda línea de costa) es testimonial.

Como consecuencia de la dinámica descrita, el territorio se ha convertido en un espacio continuo e interconectado, que se asimila a una ciudad deconstruida (Moreira, 2012), ya que ha perdido en gran medida su estructura y coherencia interna, 
caracterizándose por la existencia de núcleos poblacionales aislados entre sí desde un punto de vista espacial, pero comunicados por un denso entramado de carreteras. No obstante, hay unas notables diferencias entre las vías principales (nacionales y autopista) y las secundarias y de acceso a algunas urbanizaciones en entornos rurales, que son, a pesar de las mejoras, deficientes, ya que en muchas ocasiones se han superpuesto a la red de caminos rurales existente. Se trata de comunicaciones que, a pesar de que han mejorado con el paso del tiempo, en muchas ocasiones son incapaces de manejar el tráfico que se acumula, sobre todo en la época estival.

\section{Conclusiones}

El crecimiento de la población en el litoral de Alicante ha sido motivado en gran medida por la llegada de mano de obra procedente de otras regiones españolas, pero sobre todo de extranjeros, a raíz de la actividad turística, que ha ido cobrado protagonismo en esta región a partir de la década de 1960. Tal proceso se ha visto acentuado desde mediados de la década de los noventa, coincidiendo con un intenso boom en la construcción. La llegada de contingentes de población con rasgos específicos (jubilada o prejubilada, procedente del centro y norte de Europa) y atraída en gran medida por la benignidad climática, viviendas baratas y unos servicios aceptables, va a generar significativas repercusiones desde el punto de vista territorial (cambios en los usos del suelo, difusión de modelos residenciales de baja y media densidad, aparición de urbanizaciones segregadas del núcleo de población tradicional, entre otros) y socioeconómicas (cambio en las funciones productivas del suelo, desarrollo de servicios y actividades económicas específicas para esta población). La difusión de los usos turístico-residenciales con tipologías urbanas extensivas o semiextensivas y el incremento significativo de la población extranjera son algunos de los rasgos más destacados. La población extranjera se ha incrementado notablemente, superando, incluso, a la española. Representativos son los municipios de Rojales y San Fulgencio, donde los primeros suponen más del 70\% del total de la población. Es más, incluso hay más habitantes, por ejemplo, del Reino Unido (6.262) que espańoles (2.778) en la localidad de San Fulgencio.

Las transformaciones territoriales asociadas a la difusión de usos turístico-residenciales, vinculadas a la adquisición de viviendas mayoritariamente por residentes jubilados o prejubilados procedentes del centro y norte de Europa, han provocado, asimismo, la aparición de una serie de conflictos, entre los que cabe citar el incremento de la competencia por el uso del suelo, el aumento de los consumos de determinados recursos (electricidad y agua) y de generación de residuos (basuras o aguas negras), o la segregación social. El cambio en los usos del suelo se traduce en la sustitución de los agrarios por los residenciales. La competencia entre ambas actividades económicas se va a decidir a favor de esta última, como consecuencia de las elevadas plusvalías asociadas al cambio de uso del suelo. En esta región, el espacio litoral deja de ser un factor de producción para convertirse en objeto de consumo. El incremento de las demandas hídricas y de los consumos energéticos se asocia, por un lado, al aumento del parque de viviendas, pero también al propio modelo extensivo, en el que proliferan los usos externos (jardines y piscinas), y a su carácter 
difuso, que acentúa los consumos y las pérdidas de la red. El gasto energético se vincula al mayor número de residencias, sin olvidar el incremento del consumo de combustible por movilidad, derivado de un modelo basado en la dispersión y en la concentración de funciones por motivos de trabajo, de ocio o de compra (Conselleria de Vivienda, 2012).

La segregación social y la pérdida de la identidad cultural de la población con su entorno por la no identificación con los espacios cotidianos, es otra de las consecuencias del intenso proceso urbanizador. Ambos procesos se relacionan con la llegada de elevados contingentes poblaciones procedentes del centro y norte de Europa, que adquieren viviendas en urbanizaciones construidas al margen del núcleo urbano tradicional, configurándose como islas en entornos rurales y donde la población española es testimonial (Huete \& Mantecón, 2011). La rápida transformación registrada en algunos de estos municipios en poco más de una década determina la génesis de los denominados "no lugares", espacios homogéneos y que han perdido la mayor parte de los símbolos de identidad. Esta fragmentación y segregación del territorio se acentúa por el hecho de que los núcleos residenciales disponen de una serie de servicios vinculados exclusivamente a esta población extranjera (centros comerciales, sanidad, ocio, empresas de servicios, etcétera), por lo que la vinculación con las actividades comerciales y de servicio del núcleo de población tradicional es escasa.

\section{Referencias bibliográficas}

Baños, C. J. (1999). Modelos turísticos locales. Análisis comparado de dos destinos de la Costa Blanca. Investigaciones Geográficas, (21), 35-58. Disponible en http://dialnet.unirioja. es/ejemplar/10811

Burriel, E. (2008). La década prodigiosa del urbanismo español (1997-2006). Scripta Nova, Revista Electrónica de Geografía y Ciencias Sociales, $12(270$ [64]). Disponible en http:// www.ub.es/geocrit/sn/sn-270/sn-270-64.htm

Fernández, S. \& Barrado, D. A. (2011). El desarrollo turístico-inmobiliario de la España mediterránea e insular frente a sus referentes internacionales (Florida y la Costa Azul): un análisis comparado. Cuadernos de Turismo, (27), 373-402.

Hernández, M., Rico, A. M. \& Juárez, C. (2010). Conflicts over water and land use on the coastline of the region of Valencia: agriculture versus the urban-tourist city. En C. A. Brebbia, S. Hernández \& E. Tiezzi (Eds.), The sustainable city vi. Urban regeneration and sustainability (pp. 405-417). Southamton:wit Press.

Huete, R. \& Mantecón, A. (2010). Los límites entre el turismo y la migración residencial. Una tipología. Papers, 95(3), 781-801. doi: http://dx.doi.org/10.5565/rev/papers/ v95n3.95

Huete, R. \& Mantecón, A. (2011). Más allá del turismo: movilidad residencial europea y nuevos núcleos urbanos. Boletín de la Asociación de Geógrafos Españoles, (56), 111-128. Disponible en dialnet.unirioja.es/descarga/articulo/3722317/1.pdf 
Huete, R., Mantecón, A. \& Mazón, T. (2008). ¿De qué hablamos cuando hablamos de turismo residencial? Cuadernos de Turismo, (22), 101-121. Disponible en http://revistas.um.es/ turismo/article/view/48091

Mantecón, A. (2012). El nexo entre urbanización y turismo. Análisis de la opinión pública. Papers, 97(1), 249-272. Disponible en http://www.raco.cat/index.php/Papers/article/ view/248514

Monreal, J. (Dir.) (2001). Un nuevo mercado turístico: jubilados europeos en la región de Murcia. Murcia: Universidad de Murcia.

Moreira, J. M. (2012). Urbanismo expansivo: de la utopía a la realidad. Reflexiones desde la información ambiental. En V. Gozálvez Pérez \& J. A. Marco Molina (Eds.) Geografia: retos ambientales y territoriales: conferencias, ponencias, relatorías, mesas redondas: xxii Congreso de Geógrafos Españoles (pp. 125-163). Universidad de Alicante, 2011. Madrid: Asociación de Geógrafos Españoles (AGE).

Moreno, R., Alcalde, R. \& Lurbe, K. (2004). Emigrar en la madurez, inmigrar en la vejez: las condiciones de vida de los inmigrados jubilados extranjeros en las costas mallorquinas y catalanas. Actas del IV Congreso sobre la Inmigración en España. Universitat de Girona. CD-ROM.

O’Reilly, K. (2005). Los jubilados británicos en la Costa del Sol. En V. Rodríguez Rodríguez, M. A. Casado Díaz \& A. Huber (Eds.), La migración de europeos retirados en España (pp. 151-166). Madrid: Consejo Superior de Investigaciones Científicas (CSIC), Unidad de Políticas Comparadas (UPC).

Pack, S. D. (2006). Tourism and dictatorship: Europe's peaceful invasion of Franco's Spain. Gordonsville, VA: Palgrave.

Piqueras, J. (2012). Geografía del territorio valenciano. Naturaleza, economía y paisaje. Valencia: Universitat de Valencia.

Raya, P. \& Benítez, J. J. (2002). Concepto y estimación del turismo residencial: aplicación en Andalucía. Papers de Turisme, (31/32), 66-89.

Rico, A. M. \& Hernández, M. (2008). Ordenación del territorio, escasez de recursos hídricos, competencia de usos e intensificación de las demanda urbano-turísticas en la Comunidad Valenciana. Documents d'Anàlisi Geogràfica, (51), 79-109.

Rodríguez, V. (2004). Turismo residencial y migración de jubilados. Las nuevas formas de turismo. Almería: Instituto de Economía y Geografía y Cajamar.

Salvà, P. (2002). Foreign immigration and tourism development in Spain's Balearic Islands. En M. Hall \& A. M. Williams (Eds.), Tourism and migration: News relationships between production and consumption ( $p$ p. 119-134) Dordrecht-London: Kluwer Academic Publishers. Disponible en http://link.springer.com/chapter/10.1007\%2F978-94-0173554-4_6\#page-1

Vera, J. F. (1984). Mutaciones espaciales producidas por el turismo en el municipio de Torrevieja. Investigaciones Geográficas, (2), 115-139. Disponible en http://revistes. ua.es/ingeo/article/view/1189

Vera, J. F. (2005). El auge de la función residencial en destinos turísticos del litoral mediterráneo: entre el crecimiento y la renovación. Papers de Turisme, 37(38), 95-114. Disponible en http://rua.ua.es/dspace/handle/10045/12368 
Vera, J., Casado, J. M.a \& Ramón, A. B. (2004). Consideraciones sobre el impacto del Plan Hidrológico Nacional en el sector turístico de la provincia de Alicante. En J. Melgarejo (Ed.), Repercusiones socioeconómicas del Plan Hidrológico Nacional en la provincia de Alicante (pp. 205-267). Alicante: Fundación coepa.

\section{Documentos oficiales}

Agencia Valenciana de Turismo (2013a). Oferta turistica municipal y comarcal de la Comunidad Valenciana, 2012. Valencia: Generalitat Valenciana. Disponible en http://www. turisme.gva.es/turisme/es/files/pdf/observatorio/anuarios/La\%20Oferta\%202012def.pdf

Agencia Valenciana de Turismo (2013b). El turismo en la Comunidad Valenciana, 2012. Valencia: Generalitat Valenciana. Disponible en http://www.turisme.gva.es/turisme/ es/files/pdf/observatorio/anuarios/Turismo_CV_2012.pdf

Conselleria de Vivienda (2012). Sistema de indicadores. 2. Disponible en: http://www.cma.gva. es/web/indice.aspx?nodo $=61629$ \&idioma $=\mathrm{C}$

Exceltur (2011). Impactur 2010. Estudio del impacto económico del turismo sobre la economía de la Comunitat Valenciana. Disponible en: http://exceltur.org/excel01/contenido/portal/ files/Impactur\%20Comunitat\%20Valenciana\%202010\%20web.pdf

Instituto Nacional de Estadística (INE). (2012). Censos de población y vivienda. 1991, 2001 y 2011. Disponibles en http://www.ine.es/inebmenu/mnu_cifraspob.htm

Ministerio de Fomento (2012). Estimación del parque de viviendas, 2001-2011. Disponible en http://www.fomento.gob.es/BE2/?nivel=2\&orden=33000000 